\title{
Qualität und Integrität bei der Erstellung und Veröffentlichung wissenschaftlicher Ergebnisse
}

\author{
Lüscher, T F
}

\begin{abstract}
The publication of scientific manuscripts is an essential part in the research process and in the attempt to produce novel knowledge: only what is published exists. It is the aim of research to produce reproducible and sustainable knowledge. Reproducible knowledge is based on precise observation, the use of modern methodologies and an appropriate statistical analysis. As a consequence, it must be the intention of any scientist to report the truth and nothing but the truth. This principle requires precision and honesty. Deviation from such a behavior may lead to scientific misconduct: It encompasses the use of inappropriate methods and/or statistics, double publication of data, sloppy data presentation and processing, up to data massaging, manipulation, data theft or fabrication. Famous examples can be found throughout the history of research but it appears that such behavior has recently become more common possibly due to excessive competition, the crucial role of grants for scientific productivity and funding as well as promotion. Accordingly, in the training of researchers it seems essential to emphasize the importance of precise data acquisition and analysis to ascertain reproducible data. Similarly, it must be assured that data sets are only published once, that authors have contributed technically and/or intellectually in an important manner and that the work of other scientists is cited appropriately. Editors and reviewers should carefully assess the quality of submitted manuscripts. In fact, it is the aim of the peer review process to assure as much as possible that the quality of submitted manuscripts meets current methodological as well as ethical standards.
\end{abstract}

DOI: https://doi.org/10.1007/s00059-014-4120-3

Other titles: Quality and integrity in the production and publication of scientific results: data trimming, manipulation and (self) plagiarism

Posted at the Zurich Open Repository and Archive, University of Zurich

ZORA URL: https://doi.org/10.5167/uzh-107656

Journal Article

Published Version

Originally published at:

Lüscher, T F (2014). Qualität und Integrität bei der Erstellung und Veröffentlichung wissenschaftlicher Ergebnisse. Herz, 39(5):551-557.

DOI: https://doi.org/10.1007/s00059-014-4120-3 
Herz 2014 · 39:551-557

DOI 10.1007/s00059-014-4120-3

Online publiziert: 26. Juni 2014

(c) Urban \& Vogel 2014
T.F. Lüscher

Klinik für Kardiologie, Universitäres Herzzentrum, Universitätsspital Zürich, Zürich wissenschaftlicher Ergebnisse

\author{
Daten-Trimming, -manipulation, \\ und (Auto-)Plagiate
}

\begin{abstract}
Nur was veröffentlicht wird, existiert daher die Bedeutung von Publikationen, in der Wissenschaft, in der Kultur und darüber hinaus. Was veröffentlicht wird, sollte wahr sein, denn nur dann kann es für die Leser nützlich sein. Entsprechend beruht der Kodex der Wissenschaft auf Ehrlichkeit, Genauigkeit und Wahrheit [1].
\end{abstract}

\section{Das Wesen des \\ Erkenntnisprozesses}

Forschung kann nur weitergelangen, wenn das von einem einzelnen Wissenschaftler - heute eher von einer Forschungsgruppe - Entdeckte für Andere nachvollziehbar und reproduzierbar ist; denn nur dann kann etwas den Test der Zeit bestehen, das Wissen der Menschheit weiterbringen und praktisch nutzbar gemacht werden. Seit Sir Karl Poppers bedeutendem Werk [2] wissen wir, dass es nicht die Verifikation sein kann, die Wissen schafft, vielmehr ist es die Falsifikation, die das Unwissen vom Wissen trennt - was übrig bleibt, was bestand hat, dürfen wir Erkenntnis nennen.

Forschung hangelt sich also von Hypothese zu Hypothese und versucht sich an der Wirklichkeit zu bewähren („,onjectures and refutations" [3]). Annahmen, die der Falsifikation entgehen, machen unser Wissen aus. Das erklärt, weshalb die meisten wissenschaftlichen Erkenntnisse falsch sind, den Test der Zeit nicht bestehen [4]. Das entwertet Befunde nicht grundsätzlich, es entspricht einfach dem Erkenntnisprozess. Dass man widerlegt wird, darf in keinem Fall mit Betrug gleichgesetzt werden; der Falsifikation erliegen vielmehr die meisten Erkenntnisse, wie die Geschichte zeigt. Der Medikamentenentwicklung ergeht es nicht anders: Nur jedes zehntausendste Molekül schafft es zum Medikament.

\section{Das Ziel der Forschung}

Das Ziel der Forschung ist die anhaltende und nachhaltige Produktion überprüfbaren Wissens. Überprüfbares Wissen gründet sich auf eine genaue Beobachtung, nachvollziehbare Methoden, dem Prinzip der Wiederholbarkeit und auf eine angemessene statistische Analyse [5]. Entsprechend muss es das Anliegen jedes Forschers sein, die Wahrheit und nichts als die Wahrheit zu suchen und zu berichten.

Dabei ist der Wille zur Wahrhaftigkeit und nicht das Ergebnis, über das berichtet wird, entscheidend. Wie bereits Immanuel Kant in seinem einflussreichen Werk „Kritik der praktischen Vernunft“ aus dem Jahre 1788 festgehalten hat [6], gibt es nichts, das unzweifelhaft gut ist, außer einem guten Willen. Ein Forscher kann sich irren (und wir alle halten dies für ausgesprochen menschlich; errare humanum est), entscheidend bleibt aber, dass man sich mit gutem Willen irrt und nicht mit der Absicht zu täuschen. Und gewiss: Derjenige, der nie einen Fehler gemacht hat, hat auch niemals etwas entdeckt. Betrug ist zwingend an die Absicht $\mathrm{zu}$ täuschen gebunden.

\section{Abwege der Forschung}

Nicht immer verläuft die Forschung, wie sie sollte; in der Tat ist es in der Forschungsgeschichte wiederholt zu Betrug, Fälschungen und Plagiaten gekommen [7]. Betrug ist ein hartes Wort - es kann Institutionen schädigen, Forschungsprogramme zum Stillstand bringen sowie Karrieren und Personen zerstören. Wenn wir es also gebrauchen, sollten wir dies mit Vorsicht tun; wir sollten uns bewusst sein, was wir damit meinen.

In der Tat ist das Spektrum wissenschaftlichen Fehlverhaltens breit: Es umfasst die Verwendung falscher Methoden und statistischer Analysen, Doppelpublikationen eigener Resultate, nachlässige Datendarstellung und -erhebung, Daten-

Dieser Artikel nimmt Bezug auf eine frühere Publikation des Autors [1] und aktualisiert die vorgängige Veröffentlichung. 


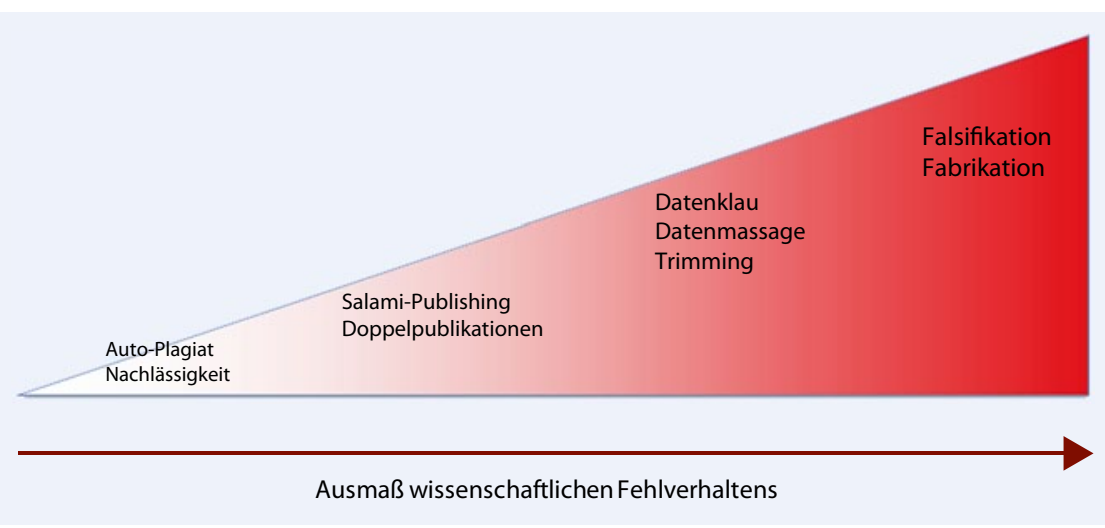

Abb. $1 \Delta$ Spektrum wissenschaftlichen Fehlverhaltens. (Mit freundlicher Genehmigung aus [1])

unterdrückung bis zu Massaging, Manipulation, Datenklau und zum freien Erfinden von Befunden (• Abb. 1; [1]). Dabei ist nicht jedes wissenschaftliche Fehlverhalten gleich zu gewichten. Ja, einige der oben genannten Möglichkeiten werden sogar von vielen Forschern zu Recht im Alltag genutzt. So gibt es gute Gründe, um gewisse Daten auszuschließen, beispielsweise weil bei der Messung technische Probleme auftraten, Zellkulturen durch Bakterien oder Viren kontaminiert waren oder Proben verwechselt wurden. Sofern dies mit gutem Willen und in begründeter Absicht geschieht (und in der Methodik Sektion der Publikation auch angemessen erwähnt wird), ist dagegen nichts einzuwenden [5]. Fehlende Sorgfalt bei der Erstellung eines Manuskripts mit beispielsweise verschieden Zahlen von Patienten in Tabellen und im Text, sind eine grobe Nachlässigkeit, aber nicht als schwerwiegendes Vergehen einzustufen - Gutachter werden kopfschüttelnd darauf hinweisen, aber dennoch nicht das Kind mit dem Bade ausschütten (bzw. das Paper ablehnen, sondern vielmehr um eine Klärung bitten). Wenn aber Daten bewusst entfernt werden, um beispielsweise eine Korrelation überzeugender zur Darstellung zu bringen, beginnt die Unehrlichkeit, ja die Fälschung von Daten. Desgleichen ist die Auf- und Abrundung von Zahlen, ja insbesondere die freie Erfindung von Ergebnissen das wohl schlimmste Vergehen eines Forschers. Somit kann wissenschaftliches Fehlverhalten aus ethischer Sicht ein breites Spektrum umfassen, von verzeihbaren Nachlässigkeiten bis zu unverzeihlichem Fehlverhalten.
Zuletzt ist heute auch vermehrt die Beeinflussung durch die forschende Industrie, die zuletzt auch Geld verdienen muss, um die hohen Investitionen, die es heute braucht, um ein Medikament oder Device zu entwickeln, in den Blickpunkt von Ethikern und Journalisten geraten. Hier heißt das Zauberwort Transparenz, als ob irgendetwas gewonnen wäre, wenn alle davon wissen. Für ernsthafte Forscher ist es zwingend, das zu berichten, was sie finden - the data are the data. Daran müssen wir festhalten, wenn wir die Glaubwürdigkeit der Forschung und Entwicklung erhalten wollen; das schließt gewiss nicht aus, dass erfolgreiche Produkte auch entsprechend in wissenschaftlichen Arbeiten eingestuft werden. Funding, Grants und Honorare sind gewiss auszuweisen, doch ist damit noch nicht das wirklich Wichtige an die Hand genommen: Das ausgewogene Urteil, die unabhängige Wertung der Befunde und klinisch relevante Einstufung der Daten dies ist entgegen landläufigen Auffassungen nur einem Forschungsgruppenleiter möglich, der von vielen begehrt wird, der sich die Sponsoren auszusuchen weiss $[8$, 9].

\section{Bedeutung des Problems}

Wie bedeutsam ist wissenschaftliches Fehlverhalten? Als Herausgeber, Gutachter oder als Autor oder Leiter einer Forschungsgruppe müssen wir uns mit diesem Thema befassen [1]. Es geht hier nicht nur um die Ethik der Wahrhaftigkeit als solche; in der Medizin kann wissenschaftliches Fehlverhalten auch unvorhergesehene Auswirkungen auf die klinische Praxis haben und damit zuletzt Patienten schaden, ja zu Todesfällen führen.

In der Tat wurde gerade kürzlich in diesem Zusammenhang intensiv darüber diskutiert, inwiefern die im New England Journal of Medicine [10] veröffentlichten, inzwischen angezweifelten Ergebnisse der DECREASE-Studie von Don Poldermans, der von der Erasmus-Universität fristlos wegen wissenschaftlichen Fehlverhaltens entlassen wurde [11], die Empfehlungen der European Society of Cardiology zum perioperativen Management [12] in unangemessener Weise beeinflusst hat. Vorlaute Journalisten haben bereits von Hundertausenden von Toten gesprochen, welche möglicherweise durch die - bisher weder vom Autor noch von der Untersuchungskommission oder dem Herausgeber des New England Journal of Medicine zurückgezogene - Publikation der DECREASE-Studie verursacht worden seien.

\section{Häufigkeit des Problems}

Wie verbreitet ist wissenschaftliches Fehlverhalten? Es überrascht nicht, dass es keine verlässlichen Zahlen dazu gibt; dennoch scheint wissenschaftliches Fehlverhalten häufiger zu sein, als allgemein angenommen. Fanelli et al. [13] haben darüber berichtet, dass in anonymen Umfragen $2 \%$ der Forscher zugegeben hätten, Daten frei erfunden zu haben, und ein Drittel aller Forscher habe zugegeben, andere weniger schwerwiegende Fehler wie Datenselektion, nachträgliche Änderung des Studiendesigns oder Manipulation der Ergebnisse vorgenommen zu haben. Es ist davon auszugehen, dass die Wirklichkeit die Äußerungen selbst in einer anonymen Umfrage bei Weitem übertrifft.

Was besonders beunruhigt, ist die Beobachtung, dass die Anzahl von Publikationen, welche in prestigeträchtigen medizinischen Zeitschriften zurückgezogen werden mussten, in den letzten 10 Jahren stark zugenommen hat. Von besonderem Interesse ist in diesem Zusammenhang die Tatsache, dass ein Viertel aller Manuskripte aufgrund von wissenschaftlichem Fehlverhalten zurückgezogen werden mussten, viele davon in Zeitschrif- 
ten mit hohem Impact-Faktor (• Abb. 2; $[14,15])$. Das ethische Fehlverhalten und die Irreleitung von Kollegen, welche im gleichen Gebiet arbeiten, ist das eine, eine mögliche Schädigung von Patienten durch eine ungerechtfertigte Beeinflussung von Guidelines für die tägliche Praxis ist das andere. Wie oben erwähnt, bleibt immer noch unklar, ob die für die klinische Praxis wichtige DECREASEStudie zur Verwendung von Betablockern vor Hochrisikooperationen wirklich verlässliche Daten beinhaltet - entsprechend schwierig ist es, Empfehlungen zur Verwendung von Betablockern vor Hochrisikooperationen zu geben [16]. In einem anderen Fall musste das European Heart Journal vor Kurzem eine große Multizenterstudie aus Japan zur Verwendung von Valsartan bei hohem Blutdruck aufgrund von wissenschaftlichem Fehlverhalten zurückziehen [17, 18]. Auch hier wurden die Probleme bei der Durchführung und Analyse der Studie erst entdeckt, als das Hauptmanuskript und zahlreiche Spinoff-Papers bereits veröffentlicht worden waren [19, 20, 21].

Ist also wissenschaftliches Fehlverhalten häufiger als angenommen? Obschon genaue Daten fehlen, ist zu vermuten, dass das Prestige, welches damit verbunden ist, in wissenschaftlichen Zeitschriften mit hohem Impact-Faktor zu publizieren und gleichzeitig die Schwierigkeit den Review-Prozess erfolgreich zu bestehen, dazu führt, dass nicht selten Daten getrimmt, ausgelassen oder einer Massage unterzogen werden. Die Bedeutung des Impact-Faktors für das eigene Fortkommen, somit für die Karriere, für die Erlangung von Grants und damit für das Überleben als Forscher wie auch die Preise, die einzelne Länder heute für bedeutende Publikationen verleihen, haben wohl dazu geführt, dass heute vermehrt wissenschaftliches Fehlverhalten um sich greift.

Überraschenderweise findet sich wissenschaftliches Fehlverhalten wie Datenunterdrückung, Daten-Trimming, Weglassen unliebsamer Datenpunkte und andere weniger schwerwiegende Unehrlichkeiten auch bei vielen bedeutenden Forschern der Geschichte. So haben sich auch bekannte Geistesgrößen wie Gregor Mendel (1822-1884; [22]), der

Herz 2014 · 39:551-557 DOI 10.1007/s00059-014-4120-3

(c) Urban \& Vogel 2014

\section{T.F. Lüscher \\ Qualität und Integrität bei der Erstellung und Veröffentlichung wissenschaftlicher Ergebnisse. Daten-Trimming, -manipulation, und (Auto-)Plagiate}

\section{Zusammenfassung}

Das Publizieren wissenschaflticher Manuskripte ist ein entscheidender Schritt im Forschungprozess und zur Gewinnung neuer Erkenntnisse - nur was veröffentlicht wird, existiert. Das Ziel der Forschung ist die anhaltende und nachhaltige Produktion überprüfbaren Wissens. Überprüfbares Wissen muss sich auf eine genaue Beobachtung, nachvollziehbare Methoden der Datengewinnung, das Prinzip der Wiederholbarkeit und auf eine angemessene statistische Analyse stützen. Entsprechend muss es das Anliegen jedes Forschers sein, die Wahrheit und nichts als die Wahrheit zu suchen und zu berichten. Dieses Prinzip erfordert Genauigkeit und Ehrlichkeit. Abweichungen von diesem Vorgehen werden als wissenschaftliches Fehlverhalten bezeichnet: Es umfasst die Verwendung falscher Methoden und statistischer Analysen, Doppelpublikationen eigener Resultate, nachlässige Datendarstellung und -erhebung, Datenunterdrückung bis zum Massaging, Manipulation, Datenklau und das freie Erfinden von Befunden. Bekannte Beispiele finden sich in der gesamten Forschungsgeschichte; es scheint aber, dass Fehlverhalten bis zum Betrug in den letzten Jahrzehnten möglicher- weise aufgrund des hohen Konkurrenzdrucks sowie der teilweise existenziellen Bedeutung wissenschaftlicher Produktivität für Forschungs-Grants, den eigenen Lohn und das berufliche Weiterkommen zugenommen haben. Entsprechend ist es in der Aus- und Weiterbildung von Forschern entscheidend, auf die Bedeutung reproduzierbaren Wissens und die Wichtigkeit einer genauen Datenerhebung, -analyse und -darstellung hinzuweisen. Ebenso gilt es sicherzustellen, dass Daten nur einmal veröffentlicht werden, Autoren einen technischen oder intellektuellen Beitrag geleistet haben und die Ergebnisse anderer Forscher angemessen zitiert werden. Herausgeber und Gutachter haben die Qualität wissenschaftlicher Manuskripte entsprechend zu beurteilen. Es ist der Sinn des „Peerreview"-Prozesses, so weit wie möglich sicherzustellen, dass die Qualität wissenschaftlicher Manuskripte modernen methodischen und ethischen Ansprüchen genügt.

Schlüsselwörter

Genauigkeit · Ehrlichkeit · Betrug ·

Datenanalyse und Reporting · Plagiate

\section{Quality and integrity in the production and publication of scientific results. Data trimming, manipulation and (self) plagiarism}

\section{Abstract}

The publication of scientific manuscripts is an essential part in the research process and in the attempt to produce novel knowledge: only what is published exists. It is the aim of research to produce reproducible and sustainable knowledge. Reproducible knowledge is based on precise observation, the use of modern methodologies and an appropriate statistical analysis. As a consequence, it must be the intention of any scientist to report the truth and nothing but the truth. This principle requires precision and honesty. Deviation from such a behavior may lead to scientific misconduct: It encompasses the use of inappropriate methods and/or statistics, double publication of data, sloppy data presentation and processing, up to data massaging, manipulation, data theft or fabrication. Famous examples can be found throughout the history of research but it appears that such behavior has recently become more common possibly due to excessive competition, the crucial role of grants for scientific productivity and funding as well as promotion. Accordingly, in the training of researchers it seems essential to emphasize the importance of precise data acquisition and analysis to ascertain reproducible data. Similarly, it must be assured that data sets are only published once, that authors have contributed technically and/or intellectually in an important manner and that the work of other scientists is cited appropriately. Editors and reviewers should carefully assess the quality of submitted manuscripts. In fact, it is the aim of the peer review process to assure as much as possible that the quality of submitted manuscripts meets current methodological as well as ethical standards.

\section{Keywords}

Stringency · Honesty · Fraud · Data analysis and reporting $\cdot$ Plagiarism 


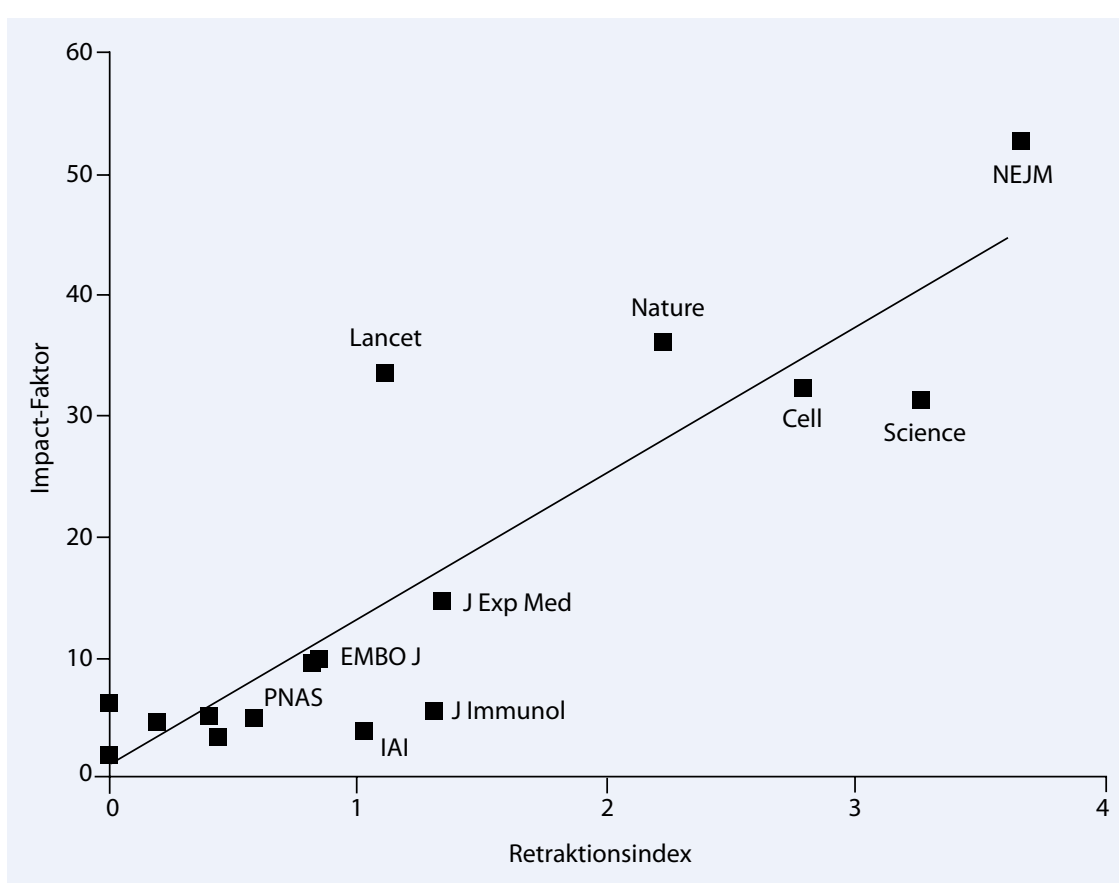

Abb. $2 \Delta$ Beziehung zwischen Impact-Faktor eines Journals und Retraktionen von Manuskripten. (Mit freundlicher Genehmigung aus [15])

die Grundlagen der modernen Genetik schuf, aber auch Charles Darwin (18091882; [23]), der Vater der Evolutionstheorie, und selbst Isaac Newton (1642-1726; [24]), der Entdecker der Gravitation und Begründer der modernen Physik geringgradige Forschungssünden zu Schulden kommen lassen. Auch wenn diese Pioniere der Wissenschaftsgeschichte Bedeutendes geleistet und zuletzt Recht hatten, konnten auch sie der Versuchung nicht ganz widerstehen, durch eine optimierte Darstellung ihrer Befunde die Zeitgenossen zu überzeugen.

Der Enthusiasmus des Entdeckers, ein unerschütterlicher Glaube an die eigene Hypothese oder Theorie kann nicht nur $\mathrm{zu}$ großen Entdeckungen führen, sondern auch die Sichtweise verengen. Das kann auch ohne Täuschungsabsicht Forscher dazu verleiten, Daten ihren Erwartungen anzupassen, zu massieren oder eben „Ausreißer“, die nur die Normalverteilung von Befunden wiedergeben, herauszunehmen. Damit können „Signifikanzen" entstehen, die nicht der Wirklichkeit entsprechen.

Auch in neuster Zeit kam es wiederholt zu Forschungsskandalen in der Dermatologie, der Immunologie und jüngst auch in der Psychologie und in ande- ren Forschungsgebieten. In der Kardiologie wurde in den 80er-Jahren der Fall von John Darsee, einem intellektuell brillanten und einnehmenden jungen Mann, weltweit bekannt, der bereits im Alter von 33 Jahren 133 Publikationen, darunter auch im New England Journal of Medicine, in Circulation und in weiteren prestigeträchtigen Zeitschriften, ausweisen konnte. Aufgrund seiner erfolgreichen Forschungstätigkeit an der Emory University wurde er alsbald von Eugene Braunwald nach Harvard berufen, wo er wiederum in kürzester Zeit eine beeindruckende Produktivität entfalten konnte. Eines Tages allerdings fiel einem Kollegen im Cardiac Research Laboratory am Brigham and Women's Hospital in Boston auf, dass Darsee Druckkurven offensichtlich falsch beschriftete. Der Kollege meldete dies Robert A. Kloner, dem Leiter des Forschungslabors, und dieser informierte umgehend den Departementschef Eugene Braunwald [25]. Zuerst glaubten alle, dass Darsee - wie dieser selbst verzweifelt versicherte - nur aufgrund des Zeitdrucks für die Einreichung eines Abstracts bei der American Heart Association einmalig die rote Linie des wissenschaftlichen Kodex überschritten hatte; bald zeigte sich aber, dass fast al- le von Darsee erhobenen Ergebnisse manipuliert oder frei erfunden waren [26].

\section{Was darf mehrmals publiziert oder von anderen Autoren übernommen werden?}

Gerade in Deutschland, aber als Folge auch in der Schweiz, ist der Plagiatsvorwurf zur neuen Waffe politischer Gegner und frustierter Kollegen geworden - entsprechend vorsichtig müssen wir in der Beurteilung solcher Vorwürfe sein. Es ist zweifellos politisch unklug und moralisch inakzeptabel, wenn ein prominenter Politiker, wie beispielsweise Karl-Theodor zu Guttenberg, sich spät in seiner Karriere eine Dissertation durch Ghostwriter verfassen lässt und dabei übersieht, wie sie zusammenkopiert wurde. In den Geisteswissenschaften sind allerdings, wie das Beispiel der Bundesministerin Annette Schawan zeigt, die Grenzen zwischen Zitat, Beeinflussung durch Gedanken der Literatur, Übernahme der Fachsprache und von Ausdrücken wichtiger Autoren des behandelten Fachgebiets und Plagiat fließend; ja, in den Geisteswissenschaften wird meist die vorhandene Literatur eingehend studiert und diskutiert, ohne dass im engeren Sinne eigene Daten erhoben werden. Dass dabei der Autor, je nach Situation, gelegentlich die Sprache der bedeutendsten Autoren des Interessengebiets teilweise oder gar (zu) deutlich übernimmt, lässt sich nachvollziehen, wenn man es auch nicht unbesehen gutheißen darf. Wir erinnern uns: Der Ausdruck „Plagiat“ kommt vom lateinischen „plagiārius" für „Seelenverkäufer, Menschenräuber"; heute ist damit im übertragenen Sinne die undeklarierte Anmaßung fremder geistiger Leistungen gemeint. Am besten entgeht man also dem Vorwurf, wenn man den- oder diejenigen, die einen inspiriert haben, wo immer möglich zitiert.

Ganz anders aber die Lage in den $\mathrm{Na}-$ turwissenschaften: Eine wissenschaftliche Arbeit berichtet hier über Daten, die man selbst (und niemand anders) erhoben hat und die nur einmal zu veröffentlichen sind. Einmal in der „public domain“, können sie zwar zitiert, aber auch vom Autor selbst nicht mehr als Originaldaten verwendet werden - und dies 
gilt ebenso für Text wie für Tabellen und Abbildungen. Werden sie - beispielsweise in einer Übersicht - erneut verwendet, muss die Originalarbeit entsprechend zitiert werden.

Beliebt ist allerdings weiterhin das „Salami-Publishing“(• Abb. 1). Gewisse Multizenterstudien, wie beispielsweise die LIFE-Studie [27] zur Wirkung von Losartan im Vergleich zu einem Betablocker bei Patienten mit arterieller Hypertonie, haben Tausende Spin-off-Papers hervorgebracht. Wie weit dürfen wir dabei gehen? Zweifellos lassen sich nicht alle Daten und Analysen einer Multizenterstudie, vor allem bei den Längenbeschränkungen für Manuskripte in den besten Zeitschriften, in einem Artikel unterbringen. Vordefinierte Subanalysen sind gewiss problemlos, ja sie müssen zur Veröffentlichung gelangen. Je mehr Subanalysen allerdings gemacht werden, desto eher finden sich signifikante Ergebnisse, die sich nicht reproduzieren lassen. Berühmt ist die Subanalyse der ISIS-2-Studie nach Sternzeichen [28] - es gibt also hier eine Grenze seriöser Forschungsarbeit!

\section{Wer ist ein Autor?}

Anders als zu William Harveys Zeiten wird heute Forschung nicht von Einzelpersonen, sondern von Forschergruppen, manchmal auch von Forschungsnetzwerken betrieben. Entsprechend kam es in den letzten Jahrzehnten zu einem steten Anwachsen der Autorenzahl, insbesondere in Multizenterstudien in der Medizin und in physikalischen Großprojekten. Gelegentlich ist es schwierig auszumachen, wer am meisten zu den Befunden beitrug - daher die „shared first authorships“ und die „shared last authorships“.

$\mathrm{Zu}$ beachten ist: Für ein erfolgreiches Projekt braucht es Ideen, ein laufendes Labor oder logistische Strukturen (welche meist der „senior author" oder der Laborleiter über Jahre aufgebaut hat), Datengewinnung, etablierte experimentelle Modelle und Methoden (für die das Gleiche gilt), Analysen und zuletzt ein wohlgeschriebenes Manuskript. Meist vereinen einzelne Forscher nicht alle diese Fähigkeiten auf sich, sodass die Veröffentlichung nur im Team erfolgen kann.
Dass ein Beitrag gemacht werden muss, um genannt zu werden, steht außer Zweifel; dieser Beitrag kann aber die Idee als solche, die Forschungsarbeit, die Analyse der Daten, eine bedeutende Methode oder auch das Schreiben oder die Durchsicht des Manuskripts sein.

Politische Autorenschaften sind nicht ungewöhnlich; so verspricht man sich von berühmten Namen höhere Chancen in renommierten Journalen - grundsätzlich gelten jedoch auch hier die oben genannten Regeln. Dabei kann die Absicht bestehen, sich bei einflussreichen „key opinion leaders“ einzuschmeicheln. Dies ist durchaus eine Gefahr für bekannte Namen, die sich für eine Veröffentlichung hergeben, die sie im Detail nicht beurteilen können - ja, bekannte Fälle wissenschaftlichen Fehlverhaltens (darunter auch derjenige John Darsees) zeigen, dass nicht selten Betrüger sich bekannte Forscher aus angesehenen Institutionen als Berater und dann auch als CoAutoren holen, um ihrer Arbeit besonderen Wert zu verleihen [7] - hier gilt es, Vorsicht zu bewahren und sicherzustellen, dass man Zugang zu den Originaldaten erhalten hat und weiß, was man tut.

\section{Originalarbeiten vs. Reviews und Buchkapitel}

Anders als wissenschaftliche Originalarbeiten, die über selbsterhobene Daten berichten, fassen Reviews und Buchkapitel das gegenwärtige Wissen zusammen. Entsprechend sind sie in diesem Zusammenhang völlig anders zu beurteilen. Solche Beiträge werden gerade von den Erfolgreichsten in großer Zahl geschrieben, da viele Zeitschriften und zahlreiche Lehrbücher und Monographien das Thema behandelt zu haben wünschen. Dabei lässt sich das Rad nicht alle paar Monate neu erfinden. Desgleichen lassen sich Lehrbücher, zumal wenn sie in der 20. Auflage erscheinen, nicht jedes Mal völlig neu schreiben.

Wie lässt sich dieses Dilemma lösen? $\mathrm{Zu}$ empfehlen ist erstens, bei Reviews und Kapiteln, die auf frühere Veröffentlichungen Bezug nehmen, dies auch zu vermerken, beispielsweise mit Festellungen wie "This review updates a previous publication by the authors [1]" oder zu Deutsch „Dieser Artikel nimmt Bezug auf eine frühere Publikation des Autors und aktualisiert die vorgängige Veröffentlichung". Gewisse neue Aspekte lassen sich immer anfügen, aber gewisse Ähnlichkeiten mit früheren Veröffentlichungen eines Autors dürfen hier keinesfalls als Autoplagiate eingestuft werden sonst wäre beispielsweise auch dieser Artikel nicht entstanden [1]. Zweitens sollte versucht werden, den Text entsprechend den neuen Bedürfnissen und kürzlichen Erkenntnissen anzupassen und neue Abbildungen und Referenzen hinzuzufügen.

Noch schwieriger wird die Sachlage, wenn - wie der Autor selbst kürzlich erfahren musste -Lehrbücher in der 10. oder 20. Auflage erscheinen. Während es in gewissen Gebieten, in welchen im Vergleich zur Vorauflage ein bedeutender Fortschritt zu verzeichnen war, einfach ist, ein überarbeitetes und damit neues Kapitel zu verfassen, ist es in Bereichen schwierig, in welchen kaum etwas Neues $\mathrm{zu}$ berichten ist. Wenn die Autoren dieselben bleiben, ist die Sache einfach (doch auch hier darf nicht von einem Autoplagiat gesprochen werden). Falls neue $\mathrm{Au}$ toren dazustoßen oder andere ausscheiden, sollten die Autoren der früheren Auflage in der nächsten Auflage gebührend erwähnt werden, entweder namentlich oder als frühere Bearbeiter.

\section{Aufgabe der Gutachter und Herausgeber}

Hochrangige wissenschaftliche Zeitschriften arbeiten nach dem Peer-reviewSystem, bei welchem alle eingereichten Manuskripte 2 bis 4 Gutachtern (darunter immer auch ein statistischer Gutachter, sofern die Einreichung in Betracht gezogen wird) zur Beurteilung vorgelegt werden. Man würde also erwarten, dass dabei Ungereimtheiten entdeckt werden. Das trifft heute sicher für Doppelpublikationen (alle eingereichten Manuskripte werden einer Medline-Suche unterzogen) und unangemessene statistische Analysen zu. Einige Zeitschriften sind sogar dazu übergegangen, die Originaldaten $\mathrm{zu}$ verlangen, sodass jederzeit auch im Detail nachgerechnet werden kann, ob die berichteten Mittelwerte und Signifikanzen auch stimmen. 
Dann sollten Gutachter auch einen Plausibilitätstest machen, was häufig aber nicht geschieht. So wurde eine Arbeit von John Darsee über die Taurin-Kardiomyopathie im New England Journal of Medicine veröffentlicht, obgleich eine offensichtlich frei erfundene Abbildung über eine Familie mit einem 17-jährigen Vater, welcher erstaunlicherweise bereits 4 Kinder im Alter von 8, 7, 5 und 4 Jahren gezeugt hatte, berichtete! Gutachter sollten also aufmerksam lesen, was ihnen vorgelegt wird [7]. Kein Gutachter (sämtlich Kardiologen) hatte sich offenbar Überlegungen zur Familiengründung und den dabei erforderlichen Zeiträumen gemacht ...

Datenmanipulation ist dagegen nicht leicht zu entdecken, wobei es allerdings nun statistische Programme gibt, welche die Datenverteilung analysieren. Da der Mensch nicht einfach zufällige Zahlenreihen $\mathrm{zu}$ gestalten vermag (und meist auf oder abrundet und Zahlenpräferenzen hat), lassen sich Daten auf Plausibilität ebenfalls heute prüfen.

Zuletzt erhalten Herausgeber nicht selten anonyme oder auch offene Anschuldigungen von Kollegen, Mitarbeitern oder Lesern. Um solche schwierig zu behandelnden Anfragen zu bearbeiten, hat das European Heart Journal ein Ethics Committee ins Leben gerufen (Maarten Simoons, Kim Fox und Christian Hamm), das sich solcher Fragen annimmt.

\section{Schlussfolgerungen}

- Zusammenfassend dürfen wir nicht alles glauben, was uns im Druck vorgelegt wird - kritisches Lesen bleibt für alle wichtig, für Leser, Forschungskollegen, Gutachter und Herausgeber.

- Um die Qualität und Integrität wissenschaftlicher Arbeiten weiterhin hochzuhalten, sollten wir Folgendes beachten:

1. Doktoranden und Wissenschaftliche Assistenten brauchen einen erfahrenen Mentor, der sie beim Arbeiten anleitet, berät und überwacht.

2. Ergebnisse wissenschaftlicher Projekte sollten regelmäßig in
Forschungskonferenzen innerhalb der Gruppe besprochen und beurteilt werden.

3. Alle beteiligten Autoren sollten Manuskripte sorgfältig durchsehen, ggf. ändern und Anregungen zu ihrer Verbesserung machen.

4. Ethische Bewilligungen für experimentelle und klinische Studien, inklusive „informed consent forms" müssen in jedem Fall verfügbar sein.

5. Alle Forscher sollten zertifizierte Kurse über Good Clinical Practice, ethische Aspekte bzw. Tierversuche erfolgreich besucht haben.

6. Wissenschaftliche Institutionen müssen eine Kultur der Ehrlichkeit und Genauigkeit leben und neutrale Daten ebenso als bedeutendes Ergebnis werten wie solche, die ihre Lieblingshypothese bestätigen.

7. Der Peer-review-Prozess ist ein wichtiger letzter Schritt zur Sicherstellung der Qualität und Integrität von Daten - dazu braucht es Gutachter und Herausgeber, die ihre Aufgabe ernst nehmen. - Qualität und Integrität ist also wichtig bei der Vorbereitung und Einreichung von Manuskripten, und wir sollten alle dazu beitragen, gleichzeitig aber auch vermeiden, dass wir eine Atmosphäre des Misstrauens schaffen, denn vor allem sollte Forschung Spaß machen, denn nur dann sind wir kreativ.

\section{Korrespondenzadresse}

Prof. Dr. T.F. Lüscher

Klinik für Kardiologie, Universitäres Herzzentrum, Universitätsspital Zürich

Rämistr. 100, CH-8091 Zürich

Schweiz

thomas.luescher@usz.ch

\section{Einhaltung ethischer Richtlinien}

Interessenkonflikt. T.F. Lüscher gibt an, dass kein Interessenkonflikt besteht.

Dieser Beitrag beinhaltet keine Studien an Menschen oder Tieren.

\section{Literatur}

1. Lüscher TF (2013) The codex of science: honesty, precision and truth - and its violations. Eur Heart J 34:1018-1023

2. Popper KR (1973) Logik der Forschung. Die Einheit der Gesellschaftswissenschaften, Bd 4, 5. Aufl. Mohr/Siebeck, Tübingen

3. Popper KR (1974) Conjectures and refutations: the growth of scientific knowledge. Routledge \& Kegan Paul, London

4. Lüscher TF (2013) The bumpy road to evidence. Eur Heart J 34:3329-3335

5. Lüscher TF (2012) Good publishing practice. Eur Heart J 33:557-561

6. Kant I (1969) Kritik der praktischen Vernunft. Die drei Kritiken. Alfred Kröner, Stuttgart, S $218 \mathrm{ff}$

7. Judson HF (2004) The great betrayal. Fraud in science. Harcourt Inc., Orlando

8. Lüscher TF, Landmesser U, Ruschitzka F (2010) Standing firm - the European Heart Journal, scientific controversies and the industry. Eur Heart J 31:1157-1158

9. Lüscher TF (2001) „Conflict of interest" oder Interesse am Konflikt? Vom Umgang mit Erkenntnis und Interesse in der Medizin. Schweizerische Ärztezeitung 82:2137-2144

10. Poldermans D, Boersma E, Bax JJ et al (1999) The effect of bisoprolol on perioperative mortaility and myocardial infarction in high-risk patients undergoing vascular surgery. $\mathrm{N}$ Engl J Med 341:1789-1794

11. Erasmus MC Follow-up Investigation Committee (2012) Report on the 2012 follow-up investigation of possible breaches of academic integrity. http:// cardiobrief.files.wordpress.com/2012/10/integrity-report-2012-10-english-translation.pdf

12. Task Force for Preoperative Cardiac Risk Assessment and Perioperative Cardiac Management in Non-cardiac Surgery, European Society of Cardiology (ESC), Poldermans D et al (2009) Guidelines for pre-operative cardiac risk assessment and perioperative cardiac management in non-cardiac surgery. Eur Heart J 30:2769-2812

13. Fanelli $D$ (2009) How many scientists fabricate and falsify research? A systematic review and meta-analysis of survey data. PLoS One 4:e5738

14. Nallamothu BK, Lüscher TF (2012) Moving from impact to influence: measurement and the changing role of medical journals. Eur Heart J 33:28922896

15. Fang FC, Casadevall A (2011) Retracted science and the retraction index. Infect Immun 79:38552859

16. Lüscher TF, Gersh B, Landmesser U, Ruschitzka F (2014) Is the panic about betablockers in perioperative care justified? Eur Heart J, pii: ehu056, doi:10.1093/eurheartj/ehu056

17. Yui Y (2012) Concerns about the Jikei Heart Study. Lancet 379:e48

18. Shimokawa $H$ (2012) Urgent announcement from the editor-in-chief concerning article retraction. Circ J, http://www.j-circ.or.jp/english/circulation_ journal/retraction_2012.html

19. Shiraishi J, Sawada T, Koide M et al (2012) Cardiocerebrovascular protective effects of valsartan in high-risk hypertensive patients with coronary artery disease (from the Kyoto Heart Study). Am J Cardiol 109:1308-1314 
20. Irie H, Shiraishi J, Sawada T et al (2012) WITHDRAWN: Cardio-cerebrovascular protective effects of valsartan in high-risk hypertensive patients with overweight/obesity: a post-hoc analysis of the KYOTO HEART Study. Int J Cardiol, pii: S0167-S5273(12)00894-7, doi:10.1016/j.ijcard.2012.06.103

21. Amano K, Shiraishi J, Sawada T et al (2012) WITHDRAWN: Enhanced cardio-renal protective effects of valsartan in high-risk hypertensive patients with chronic kidney disease: a sub-analysis of KYOTO HEART Study. Int J Cardiol, pii: S01675273(12)00090-3, doi:10.1016/j.jijcard.2012.01.072

22. Tanner W (1984) Gregor Johann Mendel: Leben, Werk und Wirkung. Biologie in unserer Zeit 14:8487
23. Darwin C (1872) The Expression of the emotions in man and animals. John Murray, London

24. Westfall RS (1980) Never at rest - a biography of Isaac Newton. Cambridge University Press, Cambridge

25. Lee TH (2013) Eugene Braunwald and the rise of modern medicine. Harvard University Press, Cambridge, S 285-292

26. Braunwald $E$ (1987) On analysing scientific fraud. Nature 325:215-216

27. Dahlöf B, Devereux RB, Kjeldsen SE et al (2002) Cardiovascular morbidity and mortality in the Losartan Intervention For Endpoint reduction in hypertension study (LIFE): a randomised trial against atenolol. Lancet 359:995-1003
28. ISIS-2 (Second International Study of Infarct Survival) Collaborative Group (1988) Randomised trial of intravenous streptokinase, oral aspirin, both, or neither among 17,187 cases of suspected acute myocardial infarction: ISIS-2. Lancet 332:349-360

\section{Hier steht eine Anzeige.}

\section{算 Springer}

